THE causative agent of acquired immune deficiency syndrome or AIDS (variously known as LAV, HTLV-III or ARV virus) is akin to the lentiviruses'. It would appear, on current estimates of AIDS virus prevalence in the US population (about 0.5 per cent), that between $10^{5}$ and $10^{6}$ persons may develop lentivirus-like encephalopathies during the next fifteen years. When other animals are infected with similar lentiviruses (maedi-visna virus in sheep), the disease is progressive and fatal, with an incubation period of one to six years. The duration of AIDS encephalopathies already known in humans is not well established but may range from one to 14 years.

The similarities between the two diseases and their causative agents are established experimentally. Both of them, RNA retroviruses, are integrated into the genome of the host's cells as proviral DNA. Efforts to treat the viral DNA must include protection for the host's DNA, a task of which we are at present incapable. Both AIDS virus and the lentiviruses possess the properties of "antigenic drift", lessening the likelihood of a vaccine as we now understand vaccines. Thus, the burden of disease control falls on epidemiology and prevention.

The implications of such a large number of men, women and children with progressive disease for the economy, health care system and emotional state of society are unprecedented. The scientific necessities, the need to develop strategies for dealing with a new type of human infection and producing a lifetime viraemia pose scientific puzzles of dizzying proportions. Even if my estimates of clinical neurological disease are in error by one or more orders of magnitude, the tragedy is still shocking, especially as it comes on top of the already manifest deaths due to AIDS immune suppression. If AIDS is a lentivirus disease, the immune-suppressed AIDS patients may be only the tip of the iceberg.

The threat of AIDS as a lentivirus disease should motivate politicians, public health workers and scientists to establish a

\section{Homochiral creationism}

\section{Sir-}

Cried God, making Adam from clay

In a dexterous left-handed way,

"Bring me PVED

$\Delta E_{\mathrm{pv}}$

Or I can't get it done in a day!"

HiLTON STOWELL

ERPB Laboratory,

120 Nature Creek SW,

Milledegville, Georgia 31061, USA

i. Tranter. G.E. Nature 318, 17?-173 (1985) unified plan for AIDS prevention on local, national and international scales. Since the mechanics of transmission of AIDSlentivirus are known, the great risk associated with anal intercourse, promiscuous heterosexual encounters, blood to blood contact and the use of shared drug injection paraphernalia must be explained in the most specific terms to all levels of society. Those already infected must be included in the strategy. Universal blood testing should not be ruled out. If AIDS is a lentivirus epidemic, to err on the side of caution would be prudent.

Cecil H. Fox

8708 First Avenue, M. Cottler-Fox

Silver Spring, Maryland 20910, USA

1. Seale, J.R. J. R. Soc. Med. 78, 613-615 (1985)

\section{Earnshaw's theorem}

SIR-This theorem is not often mentioned in scientific journals. It denies the possibility that an electric charge can be held stable solely under the electrostatic influence of other electric charge. It was used in a recent letter (Nature 317, 208; 1985) to refute a case put earlier by Berezin ( $\mathrm{Na}$ ture 315,$104 ; 1985)$. It is as well to keep in mind that the Reverend Samuel Earnshaw developed his theorem with an eye to the constitution of the aether as a medium comprising a structured system of electric charges, separated by what, presumably, would be regarded as a truly void state of the vacuum. The theorem fails if the charges permeate a charge plenum or continuum having a charge density, because displacement can then be subject to a linear restoring force rate, owing to interaction with this continuum.

We have now come to accept that the vacuum medium does have some rather special characteristics and a possible structure, so it is not unlikely that it comprises electric charges permeating a charge plenum, notwithstanding the Earnshaw theorem.

If this is the case, then the theorem cannot even be applied without some reservation when considering the mutual stability of charge in matter. The support for the structured vacuum is enhanced by the related theoretical derivation of the finestructure constant in terms of the geometrical features of an electrical charge system neutralized by a charge continuum. A value of the fine-structure constant in matching accord with its measurement at the level of one part in ten million has recently been reported from such analysis (Phys. Lett. 110A, 113; 1985).

H. Aspden

Department of Electrical Engineering,

The University,

Southampton SO9 $5 \mathrm{NH}, \mathrm{UK}$

\section{Price of star wars}

SIR-R. V. Harrowell (Nature 317, 470; 1985) criticized the proposed Strategic Defense Initiative on the grounds that if it is successful it will supposedly release deadly amounts of unexploded plutonium into the atmosphere.

Let us compare the radioactivity of this plutonium with that of the radioactive material already present in the air. According to that arcane source of data, The Handbook of Chemistry and Physics (66th edition), the atmosphere masses 5.136 hexillion grams, its mean relative molecular mass is 28.966 , and the molecular fraction of radon is one part per hexillion. If we combine this information we see there are 0.18 moles of radon in the atmosphere. The half-life of radon is 3.82 days and that of plutonium is 24,110 years; radion is therefore 2.3 million times as radioactive as plutonium. The equivalent of 400,000 moles (about 100 tons) of plutonium is already present in the air in the form of radon. We can compare that with the mass of 5,000 warheads at $10 \mathrm{~kg}$ of plutonium each, that is, 50 tons.

Joseph Hertzlinger

16 Warren Lane,

Jericho, New York 11753, USA

\section{Metric system}

SIR-There is a further problem to be considered in the controversy about ambiguities in metric notation, affecting the acquisition of mathematical concepts by school children.

In the days of Imperial measures, areas used to be denoted in units of square inches, square feet, etc. Such quantities were usually written in the form: " 16 square inches" or "16 sq. ins". This was naturally read by the child as "sixteen square inches", and was quite unambiguous.

Nowadays it is common to denote areas in the metric system as follows: " $16 \mathrm{~cm}^{2}$ " which is naturally read as "sixteen centimetres squared". A very understandable confusion then arises in a young mind between " 16 centimetres squared", which is just acceptable as a way of expressing 16 $\mathrm{cm}^{2}$, and "sixteen centimetres square" which can only mean $256 \mathrm{~cm}^{2}$.

The meaning of "sixteen square centimetres" is thus obscured, and is not easy to clarify in verbal terms.

The fault arises, of course, from the linguistic confusion between "square" and "squared" which employs minimal coding to represent an important difference. However this confusion is not helped by a system of notation which itself confuses units and quantities.

50 Highbury,

R.J. BIRD

Jesmond

Newcastle upon Tyne NE2 3EA, UK 